\title{
DIVERSIDADE E SAZONALIDADE DE CRISOMELÍDEOS (COLEOPTERA: CHRYSOMELIDAE) EM POMAR, NO MUNICÍPIO DE PONTA GROSSA, PARANÁ, BRASIL ${ }^{1}$
}

\author{
JULIANNE MILLÉO ${ }^{2}$, JANA MAGALY TESSEROLLI DE SOUZA², \\ IVANA DE FREITAS BARBOLA ${ }^{3}$, LUCIANO DE AZEVEDO MOURA ${ }^{4}$, \\ MARCELA BAER PUCCI ${ }^{5}$
}

\begin{abstract}
RESUMO - Este trabalho teve como objetivo levantar informações sobre os padrões de composição faunística, estrutura da comunidade e sazonalidade de Chrysomelidae num pomar localizado na região dos Campos Gerais do Paraná. Durante cerca de dois anos de amostragem, foram coletados 3.661 coleópteros e, destes, 1.103 crisomelídeos, representando 30,1\% dos besouros capturados. Eumolpinae e Galerucinae foram as duas subfamílias que apresentaram maior abundância e riqueza de espécies, sendo que as oito mais representativas no pomar foram: Eumolpinae sp. 7 e Eumolpinae sp. 15, Diabrotica speciosa, Iphimeis dives, Spintherophyta semiaurata, Colaspis sp. 1 e Colaspis sp. 2 e Syphrea sp. 1, representando 91,7\% dos indivíduos coletados. Em relação às árvores frutíferas, observou-se que o maior número de insetos foi coletado em laranjeira e tangerineira; a laranjeira teve a maior riqueza de espécies, e o caquizeiro apresentou o maior índice de diversidade. A redução considerável de crisomelídeos no segundo ciclo anual demonstra que a família tem uma oscilação temporal acentuada. A primavera foi a estação de maior abundância nos dois anos de coleta, e os fatores meteorológicos não apresentaram correlação com a abundância de crisomelídeos. Termos para indexação: Análise faunística; sazonalidade; árvores frutíferas; bioecologia; flutuação de crisomelídeos; Campos Gerais do Paraná.
\end{abstract}

\section{CHRYSOMELIDS DIVERSITY AND SEASONAL OCCURRENCE (COLEOPTERA: CHRYSOMELIDAE) IN AN ORCHARD IN PONTA GROSSA DISTRICT, PARANÁ, BRAZIL}

\begin{abstract}
This study aimed to obtain information about the fauna composition standards, community structure and Chrysomelidae seasonality in an orchard located in Campos Gerais region of Paraná. During the nearly two years of sampling, 3,361 coleopterous were collected, and among these coleopterous, 1,103 belonged to Chrysomelidae family, representing $30.1 \%$ of the captured beetles. Eumolpinae and Galerucinae subfamilies showed the biggest species abundance and richness, furthermore, the most representative to the orchard were: Eumolpinae sp. 7 and Eumolpinae sp. 15, Diabrotica speciosa, Iphimeis dives, Spintherophyta semiaurata, Colaspis sp. 1 and Colaspis sp. 2 and Syphrea sp. 1, representing 91.7\% of the collected coleopterous. In relation to the fruit trees, it was observed that the biggest insect number was collected on the orange and tangerine trees; orange tree had the biggest species richness index and persimmon tree showed the biggest diversity index. There was a considerable reduction of the Chrysomelidae species on the second annual cycle, and this fact demonstrated that the family has a great temporal variation. Spring was the season with the largest abundance on two years of collection and the meteorological factors did not show any relation to the Chrysomelidae abundance.
\end{abstract}

Index terms: Fauna analysis; seasonality; fruit trees; bioecology; fluctuation of chrysomelids; Campos Gerais do Paraná.

\footnotetext{
1(Trabalho 203-12). Recebido em: 04-07-2012. Aceito para publicação em: 07-06-2013.

${ }^{2}$ Prof ${ }^{a s}$ Associadas, Universidade Estadual de Ponta Grossa, Campus de Uvaranas, R. Carlos Cavalcanti 4748, CEP. 84030-900, Ponta Grossa/PR. E-mail: jmilleo@hotmail.com, ibarbola@yahoo.com.br.

${ }^{3}$ Doutoranda, Programa de Pós-Graduação em Ecologia e Conservação, Centro Politécnico, Universidade Federal do Paraná, Caixa Postal. 19031, CEP. 81531-980, Curitiba/PR. E-mail: janamagaly@yahoo.com.br

${ }^{4}$ Pesquisador Visitante, Fundação Zoobotânica do Rio Grande do Sul, R. Dr. Salvador França, 1427, CEP. 90690-000, Porto Alegre/ RS. E-mail: iucetima@hotmail.com.

${ }^{5}$ Mestranda, Programa de Pós-Graduação em Biologia Evolutiva, Universidade Estadual de Ponta Grossa, Campus de Uvaranas, R. Carlos Cavalcanti 4748, CEP. 84030-900, Ponta Grossa/PR. E-mail: marbaer@gmail.com.
} 


\section{INTRODUÇÃO}

Uma análise sobre a flutuação populacional da entomofauna associada a culturas de interesse econômico é de suma importância para estudos de avaliação de danos, além de fornecer subsídios para o manejo integrado de pragas (CANETTIERI; GARCIA, 2000).

Chrysomelidae é uma das cinco famílias mais numerosas de Coleoptera e a segunda maior entre os herbívoros (RILEY et al., 2002). Sua notável diversidade, associada com uma distribuição mundial e dieta fitófaga, da aos crisomelídeos considerável importância ecológica e econômica (CHABOO, 2007).

Embora existam muitas dúvidas sobre a classificação ao nível de subfamílias e tribos, segundo Bouchard et al. (2011), 12 subfamílias integram Chrysomelidae: Bruchinae, Cassidinae, Chrysomelinae, Criocerinae, Cryptocephalinae, Donaciinae, Eumolpinae, Galerucinae, Lamprosomatinae, Sagrinae, Spilopyrinae e Synetinae. Os indivíduos são predominantemente fitófagos nas formas larval e adulta, apresentando considerável especificidade na alimentação. Algumas tribos de crisomelídeos apresentam preferência alimentar por determinadas famílias botânicas: Cassidini por Convolvulaceae; Galerucini por Cucurbitaceae, e Chalepini por Poaceae e Arecaceae.

Levantamentos que avaliam os crisomelídeos em uma abordagem ecológica são escassos no Brasil. Gonçalves (2008) inventariou as espécies ocorrentes em uma reserva natural, em Viçosa-MG, determinando a relação entre o número de espécies e os fatores meteorológicos. Linzmeier e Ribeiro-Costa (2012) avaliaram a composição faunística e a estrutura da comunidade de crisomelídeos, buscando estabelecer comparações entre vários ambientes do Estado do Paraná, entre estes o Parque Estadual de Vila Velha, no município de Ponta Grossa.

Entretanto, a região dos Campos Gerais carece de informações a respeito da diversidade destes besouros associados a árvores frutíferas. Este estudo teve como objetivos: (a) determinar a composição de espécies de Chrysomelidae em um pomar em Ponta Grossa, Paraná; (b) apresentar uma análise faunística e estrutural da comunidade; e (c) verificar se há sazonalidade na flutuação de crisomelídeos durante o período de amostragem.

\section{MATERIAIS E MÉTODOS}

O estudo foi realizado no pomar do Colégio Agrícola Estadual Augusto Ribas (Ponta Grossa-PR), com área de aproximadamente $1.000 \mathrm{~m}^{2}\left(25^{\circ} 05^{\prime} 42^{\prime}\right.$ 'S, $50^{\circ} 06^{\prime} 17^{\prime}$ 'W; $900 \mathrm{~m}$ ), de setembro de 2004 a junho de 2006, totalizando 47 coletas. As coletas foram feitas quinzenalmente, no período matutino, com uso de guarda-chuva entomológico.

Foram selecionadas previamente três árvores de cada espécie do pomar (Tabela 1). Para facilitar a leitura, as referências às árvores frutíferas no texto foram feitas com base em seus frutos. Nas amostragens, somente os coleópteros foram avaliados. Padronizou-se balançar por três vezes os galhos de cada uma das árvores selecionadas, provocando a queda dos insetos sobre o guarda-chuva entomológico.

No laboratório, os crisomelídeos foram separados em morfoespécies, fixados em alfinetes entomológicos, etiquetados e, posteriormente, identificados ao menor táxon possível, adotando-se a classificação de Bouchard et al. (2011). As identificações foram baseadas em chaves dicotômicas e descrições, comparação com os exemplares da Coleção de Entomologia Pe. Jesus Santiago Moure (DZUP), Departamento de Zoologia, Universidade Federal do Paraná, Curitiba-PR e ainda com a colaboração de especialista na área. O material estudado encontra-se depositado na Coleção Entomológica dos Campos Gerais do Paraná (CECG), Universidade Estadual de Ponta Grossa, Ponta Grossa-PR.

Análises faunísticas e de estrutura da comunidade. Os dados foram analisados no programa ANAFAU, desenvolvido pela ESALQ, no qual foram avaliados os índices de frequência, abundância, dominância e constância. De acordo com os resultados, foram selecionadas as espécies predominantes, designadas como "indicadores ecológicos" e nas quais se baseou a discussão deste trabalho.

As análises listadas a seguir foram realizadas com o PAST versão 2.07. A diversidade de crisomelídeos no pomar foi calculada pelos índices de Shannon-Wiener (H') e Margalef $(\alpha)$ e, para verificar se existiu diferença significativa entre os valores de diversidade obtidos por meio do primeiro índice, foram aplicados testes $t$. A uniformidade, em termos de abundância de indivíduos, distribuída entre as espécies amostradas, foi calculada pelo índice de equitatividade (E'). Para a avaliação da similaridade entre as árvores frutíferas com relação à composição de espécies, foi aplicado o coeficiente de Jaccard (dados de presença/ausência), e com relação à estrutura da comunidade, foi aplicado o índice de Morisita (dados de abundância).

Sazonalidade. Como os valores de abundância não apresentaram distribuição normal nem variâncias homogêneas (mesmo realizando-se transformações), testes não paramétricos foram aplicados. A fim de testar se a abundância dos crisomelídeos 
apresentou variação temporal, foi realizada uma análise de Kruskal-Wallis, tendo a abundância como variável resposta e o ano de coleta e a estação sazonal como fatores. Para ajustar a análise ao período de coletas, as estações foram consideradas como se segue: inverno $\left(1^{\circ}\right.$ ano - só setembro, $2^{\circ}$ ano - de julho a setembro), primavera (de outubro a dezembro), verão (de janeiro a março) e outono (de abril a junho).

A fim de verificar a influência de fatores meteorológicos (precipitação acumulada, umidade relativa do ar, temperaturas máxima, média e mínima) na flutuação populacional ao longo da amostragem, foi realizada uma análise de correlação de Spearman entre os valores de abundância mensal total (soma de todas as plantas) e os fatores meteorológicos. Os dados climáticos foram fornecidos pelo Instituto Tecnológico SIMEPAR, Estação Meteorológica de Ponta Grossa. Todas as análises foram feitas no R 2.13.1.

\section{RESULTADOS E DISCUSSÃO}

\section{Análises faunísticas e de estrutura da comunidade}

Durante cerca de dois anos de amostragem, foram coletados 3.661 coleópteros, e destes, 1.103 crisomelídeos, representando $30,1 \%$ dos coleópteros capturados. Das 12 subfamílias reconhecidas por Bouchard et al. (2011), sete foram registradas: Eumolpinae (56,7\%), Galerucinae (39,3\%), Cassidinae $(1,4 \%)$, Chrysomelinae $(1,4 \%)$, Bruchinae $(0,8 \%)$, Criocerinae $(0,2 \%)$ e Cryptocephalinae $(0,2 \%)$ (Tabela 2). Comparando-se estes resultados aos de Linzmeier e Ribeiro-Costa (2012), embora os métodos de amostragem tenham sido distintos, Galerucinae e Eumolpinae também foram as mais abundantes em Vila Velha (Ponta Grossa); contudo, representaram $90,5 \%$ e apenas $5,8 \%$, respectivamente.

Em relação à riqueza, foram amostradas 53 espécies, sendo Galerucinae (15) a subfamília mais diversa, seguida de Eumolpinae (14), Cassidinae (9), Bruchinae (7), Chrysomelinae (5), Criocerinae (2) e Cryptocephalinae (1). Linzmeier e Ribeiro-Costa (2012) registraram 118 táxons na região de Ponta Grossa, sendo que a riqueza de Galerucinae (63 espécies) e a de Eumolpinae (15) também foram maiores em comparação às demais subfamílias, que se mostraram pouco representativas. Em Viçosa-MG, Gonçalves (2008) registrou apenas três subfamílias entre os 610 crisomelídeos coletados: Galerucinae (53), Eumolpinae (46) e Cryptocephalinae (14). A grande quantidade e variedade de representantes de Galerucinae e Eumolpinae era esperada, pois tratase das duas subfamílias mais numerosas dentro de Chrysomelidae.

Nas análises faunísticas, entre as 53 espécies coletadas, oito foram apontadas como indicadores ecológicos e organizadas em dois grupos de acordo com os resultados: (a) Superfrequentes, superdominantes, superabundantes e constantes nas coletas - incluindo Eumolpinae sp. 7 e Diabrotica speciosa (Germar, 1824); (b) Muito frequentes, dominantes, muito abundantes e constantes nas coletas - Iphimeis dives (Germar, 1824), Spintherophyta semiaurata (Lefèvre, 1875), Colaspis sp. 1, Colaspis sp. 2, Eumolpinae sp. 15 e Syphrea sp. 1.

$\mathrm{O}$ conjunto das oito espécies mais frequentes correspondeu a $91,7 \%$ dos indivíduos coletados (Tabela 2). Esta porcentagem é bem expressiva se comparada ao trabalho de Linzmeier e Ribeiro-Costa (2012), no qual as 10 espécies mais abundantes representaram $45 \%$ do total. Provavelmente, esta elevada dominância de algumas espécies esteja relacionada à forte antropização no entorno do pomar.

Outras sete espécies obtiveram resultados menos significativos: Alagoasa sp. 1, Alticini sp. 2 (frequente, dominante, comum e constante); Isotes sp. 1 (frequente, dominante, comum e acessória); Microtheca ochroloma Stål, 1860, Desmogramma bivia (Germar, 1824), Disonycha conjuncta (Germar, 1824) e Diabrotica sp. 1 (frequente, não dominante, comum e acessória) (Tabela 2).

As 38 espécies restantes foram classificadas como pouco frequentes, não dominantes, dispersas e acidentais (Tabela 2).

Entre as espécies de Galerucinae, Diabrotica speciosa representou $32,0 \%$ dos insetos amostrados no local. Foi coletada em todas as frutíferas, nas seguintes proporções: laranja $(54,7 \%)$, tangerina $(51,2 \%)$, poncã $(42,4 \%)$, limão $(39,4 \%)$, maçã $(30,4 \%)$, pera $(24,8 \%)$, pêssego $(17,0 \%)$, caqui $(12,7 \%)$ e nectarina $(11,7 \%)$ (Tabela 2$)$. Dentre os coleópteros desfolhadores, esta espécie é uma das mais importantes pragas agrícolas, sendo generalista e de ampla disseminação nos estados brasileiros e na maioria dos países da América do Sul (GALLO et al., 2002).

Spintherophyta semiaurata (Eumolpinae) foi constatada em todas as árvores, especialmente em caqui e pera, correspondendo a 29 e $19 \%$ dos crisomelídeos coletados, respectivamente. Pouco se sabe sobre a bioecologia de indivíduos deste gênero; entre os trabalhos mais recentes, destacam-se Zawadneak et al. (2011), em que $S$. semiaurata foi relatada pela primeira vez danificando flores de morangueiro (Fragaria x ananassa) no município de São José dos Pinhais-PR.

As três espécies de Colaspis encontradas representam 5,0\% dos crisomelídeos neste levantamento, estando mais associadas à nectarina, limão e caqui. Várias espécies de Colaspis têm sido observadas em Solanaceae, mas há também registros deste gênero infestando folhas de frutas, tais como bananeira, na América do Sul (JOLIVET e VERMA, 2008). 
Apesar de as árvores frutíferas se encontrarem muito próximas entre si no pomar, a maioria das espécies de crisomelídeos demonstrou preferência por algumas delas. Apenas quatro espécies foram comuns às nove frutíferas avaliadas: $D$. speciosa, Eumolpinae sp. $15, S$. semiaurata e I. dives, indicando possuírem hábito generalista.

A composição das espécies mostrou-se muito diferente da obtida por Linzmeier e Ribeiro-Costa (2012) ao se comparar as espécies mais abundantes listadas para o município de Ponta Grossa, com exceção dos representantes do gênero Syrphraea encontrados em número elevado em ambas as análises. Esta diferença, provavelmente, deve-se ao método distinto de amostragem e/ou aos tipos vegetacionais amostrados, já que Linzmeier e Ribeiro-Costa (2012) coletaram em Floresta Ombrófila Mista Montana, e o presente levantamento ocorreu em espécies frutíferas exóticas.

A respeito da abundância relativa de crisomelídeos por árvore frutífera, obtiveram-se as seguintes porcentagens: laranja e tangerina $(15,1 \%)$, nectarina $(14,8 \%)$, caqui $(13,6 \%)$, Pera $(11,7 \%)$, pêssego (10,1\%), limão $(9,9 \%)$, poncã $(7,7 \%)$ e maçã $(2,0 \%)$ (Tabela 2).

O índice de Shannon-Wiener variou de 2,174 a 1,651 entre as espécies vegetais, na ordem: caqui $>$ poncã $>$ nectarina $>$ limão $>$ maçã $>$ pêssego $>$ tangerina $>$ laranja $>$ pera (Tabela 3 ). Os valores de diversidade foram significativamente diferentes entre o caqui e as demais frutíferas (exceto a poncã); e entre a poncã e a laranja, tangerina, pera e pêssego (Tabela 4). Este índice revela que as comunidades de crisomelídeos do caquizeiro e da árvore de poncã foram as mais diversas.

Ao se comparar o índice de Shannon-Wiener do pomar $(2,249)$ ao obtido por Linzmeier e Ribeiro-Costa (2012) para a região de Ponta Grossa $(2,92)$, os valores apresentam-se aproximados. Linzmeier e Ribeiro-Costa (2012) encontraram índices variando entre 3,51 (Telêmaco Borba) e 1,68 (Antonina) para as oito localidades analisadas, sendo que os resultados aqui obtidos se encontram dentro desta faixa.

Em relação ao índice de Margalef, obteve-se a seguinte sequência: laranja $>$ caqui $>$ poncã $>$ limão $>$ tangerina $>$ nectarina $>$ pera $>$ pêssego $>$ maçã (Tabela 3), sendo que os valores variaram de 4,304 a 2,265. Já no índice de equitatividade, a variação foi de 0,9012 (maçã) a 0,5460 (laranja) (Tabela 3). Para ambos os índices calculados, mais uma vez receberam destaque o caqui e a poncã.

Não foi possível detectar agrupamentos bem definidos entre as árvores frutíferas no dendrograma, baseado no índice de Jaccard (Figura 1A). A composição de espécies de crisomelídeos mostrou-se mais similar entre limão, pêssego e caqui e entre tangerina, laranja e nectarina.
O dendrograma de similaridade obtido pelo índice de Morisita (Figura 1B) mostra que a estrutura de comunidade de crisomelídeos formou três agrupamentos distintos: o primeiro, representado pelas plantas cítricas; o segundo, contendo caqui, nectarina e pêssego; e o terceiro, somente maçã e pera. Os agrupamentos foram formados justamente por plantas que apresentam características parecidas (como a rigidez e a composição das folhas, por exemplo), o que permite maior semelhança na estrutura da comunidade dos besouros fitófagos, hospedeiros das mesmas. A maior similaridade entre a tangerina e a laranja deve-se principalmente ao elevado número de D. speciosa coletado nestas duas frutíferas.

\section{Sazonalidade}

A abundância de crisomelídeos variou significativamente entre os dois anos de coleta (Kruskal-Wallis: $\mathrm{H}=15,41 ; \mathrm{p}<0,001$ ), reduzindo consideravelmente no segundo ano, o que demonstra que a família tem uma acentuada oscilação temporal.

Os crisomelídeos apresentaram níveis de abundância sazonalmente distintos (Kruskal-Wallis: 99,51; $\mathrm{p}<0,001$ ), sendo a primavera a estação de maior número de indivíduos nos dois anos de coleta. Este dado corrobora a afirmação de outros autores para várias famílias de insetos no Paraná: Chrysomelidae (Coleoptera) (LINZMEIER; RIBEIRO-COSTA, 2008; LINZMEIER; RIBEIRO-COSTA, 2012), Muscidae (Diptera) (COSTACURTA et al., 2003) e Syrphidae (Diptera) (MARINONI et al., 2006).

Diferentemente de outros estudos, os resultados aqui obtidos não indicaram correlação entre a flutuação nos níveis de abundância e os fatores climáticos (precipitação acumulada: $\mathrm{r}=0,29$ e $\mathrm{p}>$ 0,05 ; umidade relativa do ar: $\mathrm{r}=0,36$ e $\mathrm{p}>0,05$; temperatura média: $r=0,19$ e $p>0,05$; temperatura máxima: $\mathrm{r}=0,16$ e $\mathrm{p}>0,05$; temperatura mínima: $\mathrm{r}$ $=0,18 \mathrm{e} \mathrm{p}>0,05)$. Isso indica que a sazonalidade dos crisomelídeos na área de estudo não foi determinada pelas variáveis abióticas utilizadas neste trabalho, ou que o $\mathrm{n}$ amostral não foi suficiente para detectar significância. A redução da abundância no segundo ano de coleta pode estar associada a menores valores médios de umidade relativa do ar e à precipitação acumulada no segundo ciclo anual $(78,3 \%$ e 82,5 $\mathrm{mm}$, respectivamente) em comparação ao primeiro $(84,8 \%$ e $119,5 \mathrm{~mm})$. Isso pode acontecer em razão de que tais insetos estão altamente associados à fenologia de seus hospedeiros, sendo que o período chuvoso está diretamente relacionado com o crescimento natural dos vegetais (GONÇALVES, 2008). Após as chuvas, a vegetação renova-se rapidamente, influenciando a composição das comunidades que dependem da quantidade e da variedade de massa vegetal disponível, em especial folhas e flores (GONÇALVES, 2008). 
TABELA 1- Espécies frutíferas amostradas no pomar do Colégio Agrícola Estadual Augusto Ribas, Ponta Grossa-PR, de setembro de 2004 a junho de 2006.

\begin{tabular}{ccccc}
\hline & Nome comum & Fruto/Sigla & Nome científico & Família \\
\hline \multirow{2}{*}{} & Laranjeira & Laranja (LA) & Citrus sinensis (L.) Osbeck & Rutaceae \\
Limoeiro & Limão (LI) & Citrus limon (L.) Osbeck & Rutaceae \\
Tangerineira Ponkan & Poncã (PO) & Citrus reticulata Blanco & Rutaceae \\
Tangerineira King & Tangerina (TA) & Citrus nobilis Lour. & Rutaceae \\
\hline \multirow{2}{*}{} & Caquizeiro & Caqui (CA) & Diospyros kaki Thunb. & Ebenaceae \\
& Macieira & Maçã (MA) & Malus domestica Borkh. & Rosaceae \\
& Nectarineira & Nectarina (NE) Prunus persica var nucipersica Dippel & Rosaceae \\
& Pereira & Pera (PR) & Pyrus communis L. & Rosaceae \\
Pessegueiro & Pêssego (PS) & Prunus persica (L.) Batsch & Rosaceae \\
\hline
\end{tabular}
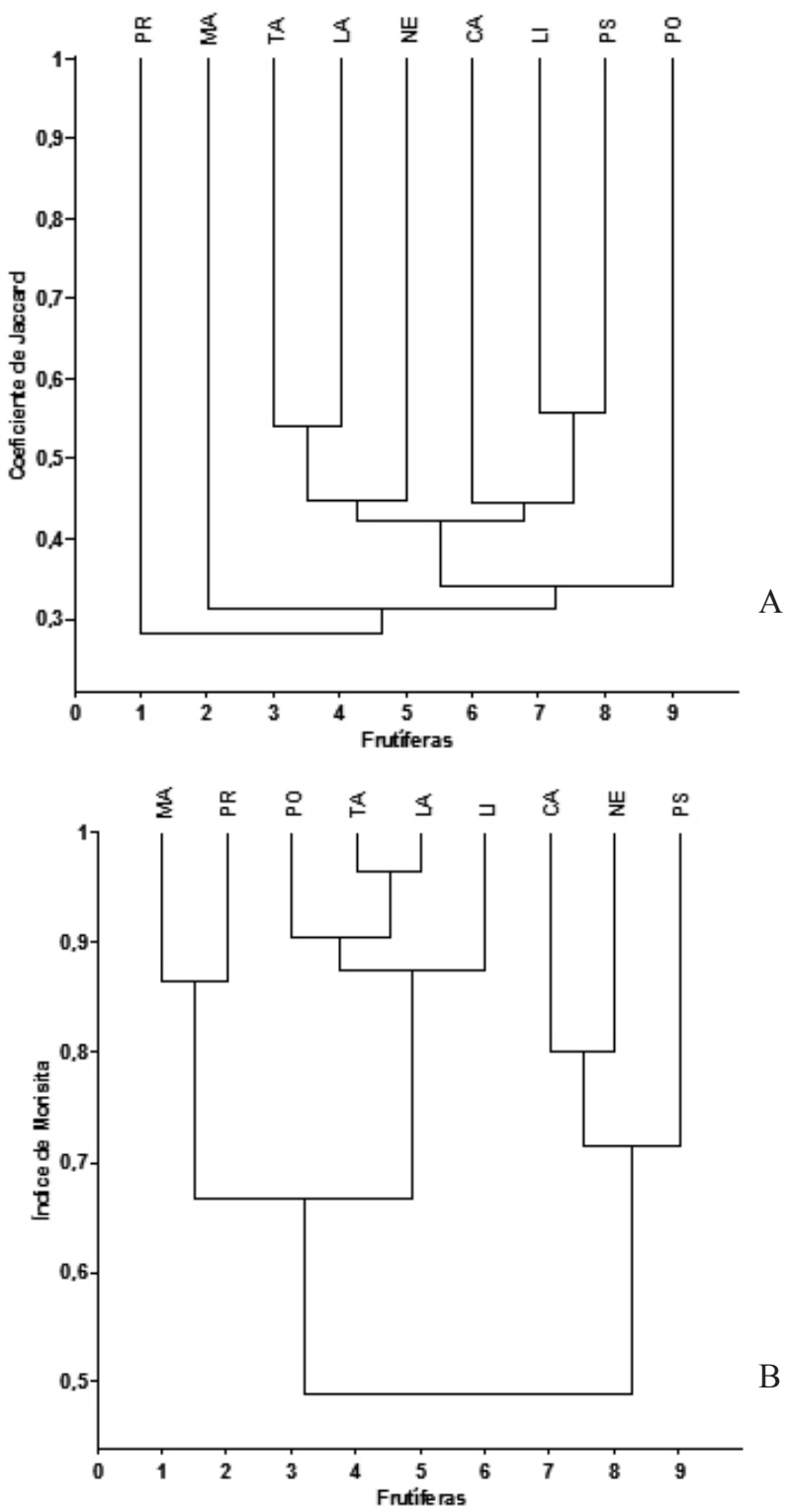

FIGURA 1- Dendrograma de similaridade da comunidade de crisomelídeos em diferentes frutíferas do pomar do Colégio Agrícola, UEPG, Ponta Grossa-PR. A) Coeficiente de Jaccard, dados de presença/ ausência. B) Índice de Morisita, dados de abundância. Laranja (LA), Limão (LI), Poncã (PO), Tangerina (TA), Caqui (CA), Maçã (MA), Nectarina (NE), Pera (PR), Pêssego (PS). 


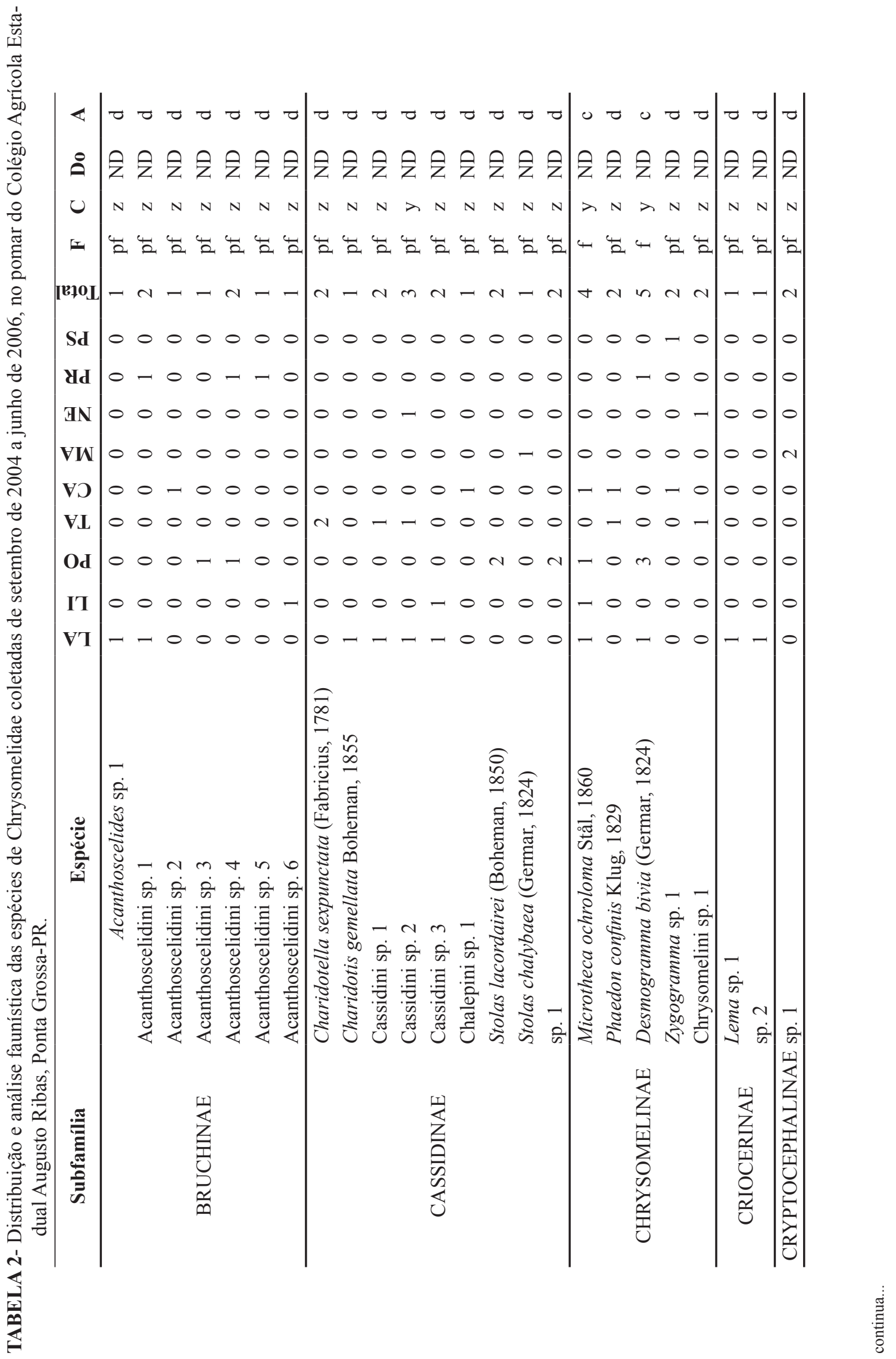




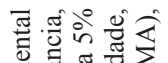

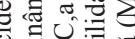

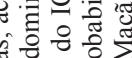

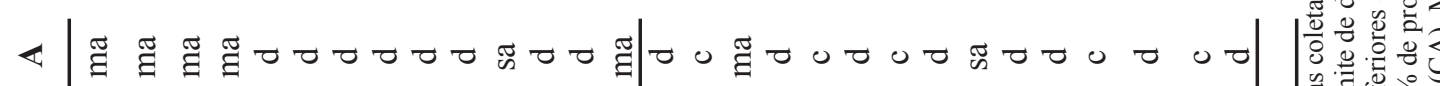

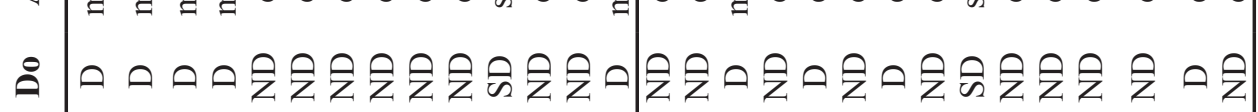

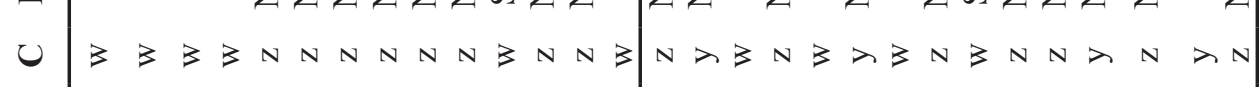

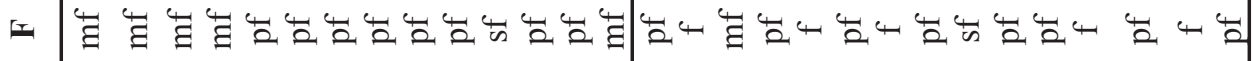

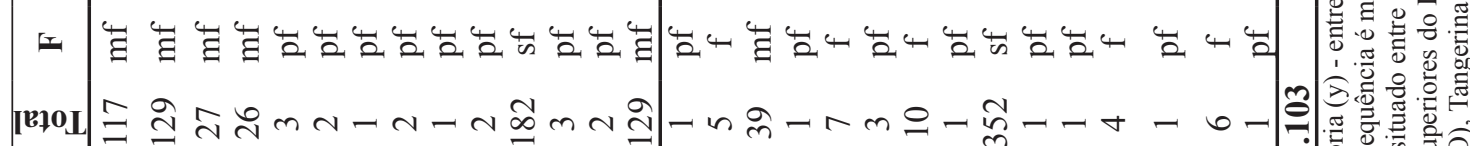

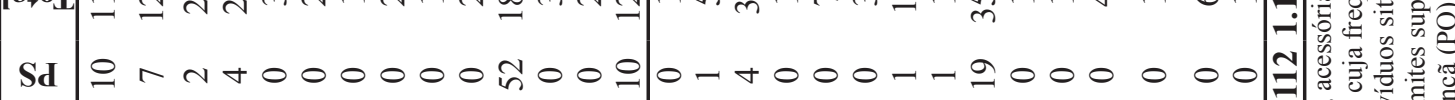
पd

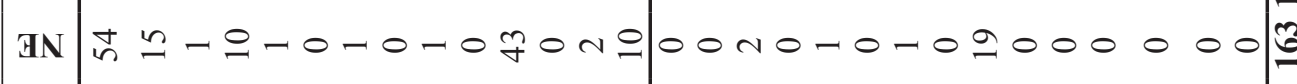
क्षे: VIN m m $00000000000 \mathrm{n} 00-000-00000000$ i VI VIL $O$ INO

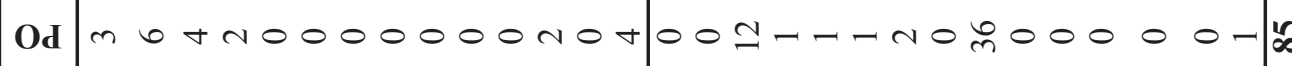

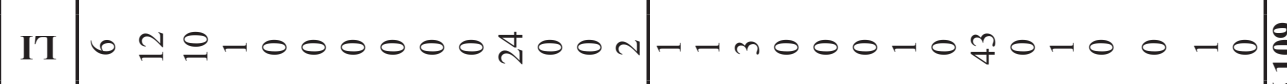

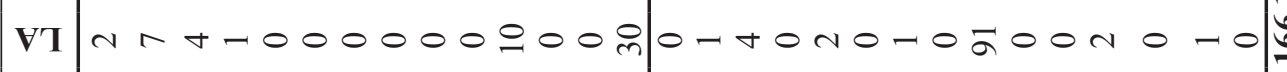
त्ञ त्व

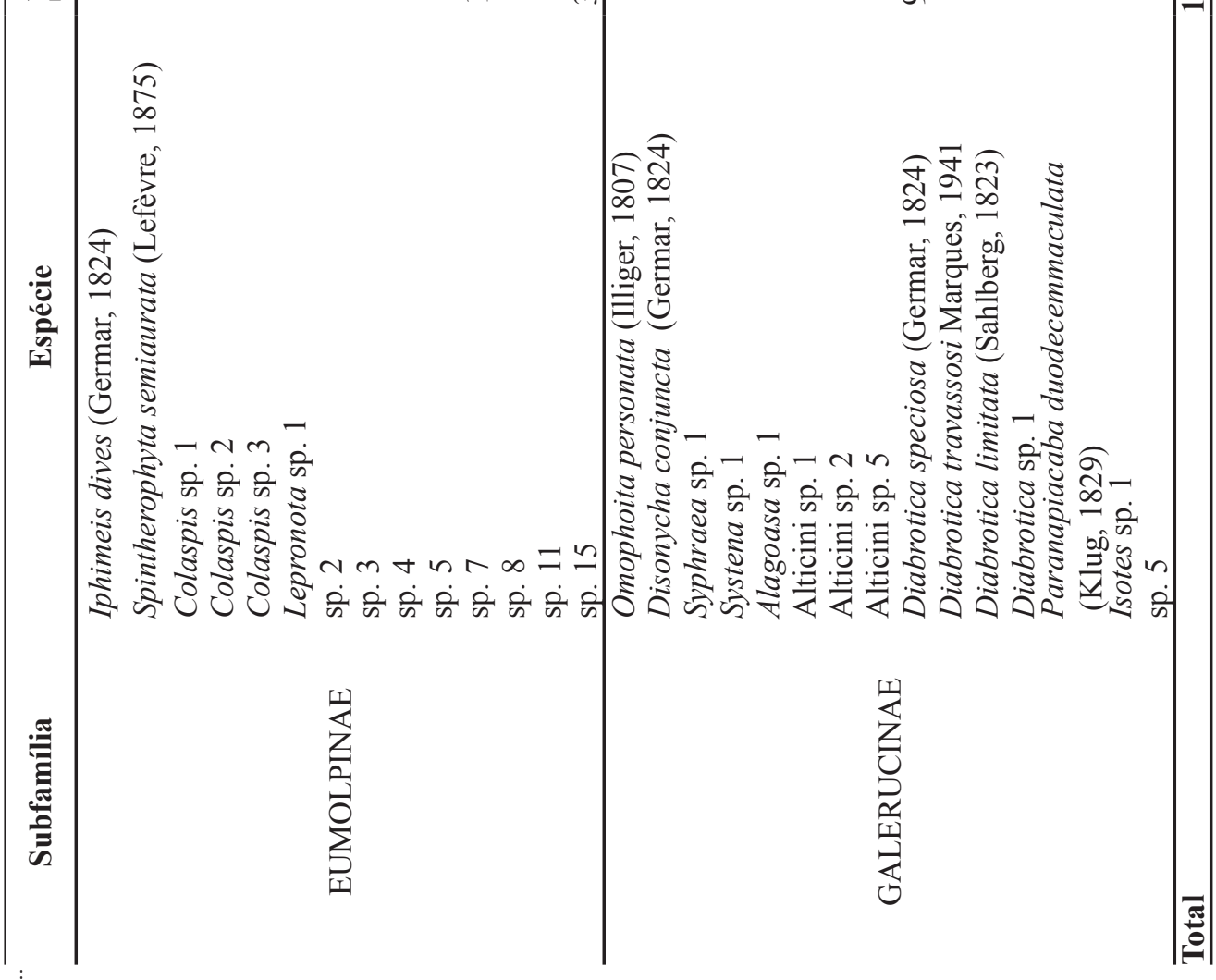


TABELA 3 - Diversidade e sazonalidade dos Chrysomelidae coletados de setembro de 2004 a junho de 2006, no pomar do Colégio Agrícola Estadual Augusto Ribas, Ponta Grossa-PR

\begin{tabular}{|c|c|c|c|c|c|c|c|c|c|c|c|c|c|c|}
\hline \multirow{2}{*}{\multicolumn{2}{|c|}{ Frutífera }} & \multicolumn{4}{|c|}{$1^{\circ}$ ano } & \multicolumn{4}{|c|}{$2^{\circ}$ ano } & \multirow{2}{*}{$\mathbf{N}$} & \multirow{2}{*}{$\mathbf{S}$} & \multirow{2}{*}{$\mathbf{H}^{\prime}$} & \multirow{2}{*}{$\alpha$} & \multirow{2}{*}{$\mathrm{E}^{\prime}$} \\
\hline & & Inv & Prim & Ver & Out & Inv & Prim & Ver & Out & & & & & \\
\hline \multirow{4}{*}{$\frac{\underbrace{n}}{\underline{v}}$} & Laranja & 5 & 15 & 28 & 59 & 21 & 14 & 14 & 10 & 166 & 23 & 1,712 & 4,304 & 0,546 \\
\hline & Limão & 2 & 54 & 22 & 18 & 1 & 2 & 8 & 2 & 109 & 16 & 1,881 & 3,197 & 0,6786 \\
\hline & Poncã & 4 & 14 & 34 & 20 & 1 & 3 & 4 & 5 & 85 & 19 & 2,158 & 4,052 & 0,7329 \\
\hline & Tangerina & 12 & 44 & 19 & 29 & 21 & 14 & 3 & 25 & 167 & 17 & 1,723 & 3,126 & 0,6083 \\
\hline \multirow{5}{*}{$\underset{\substack{0 \\
0}}{\stackrel{\infty}{0}}$} & Caqui & 0 & 100 & 9 & 8 & 5 & 23 & 5 & 0 & 150 & 22 & 2,174 & 4,191 & 0,7034 \\
\hline & Maçã & 0 & 3 & 4 & 5 & 5 & 3 & 2 & 0 & 22 & 8 & 1,874 & 2,265 & 0,9012 \\
\hline & Nectarina & 0 & 119 & 11 & 3 & 1 & 18 & 8 & 3 & 163 & 16 & 1,888 & 2,945 & 0,6810 \\
\hline & Pera & 0 & 74 & 15 & 20 & 2 & 12 & 6 & 0 & 129 & 14 & 1,651 & 2,675 & 0,6257 \\
\hline & Pêssego & 0 & 64 & 9 & 5 & 6 & 24 & 3 & 1 & 112 & 12 & 1,740 & 2,331 & 0,7003 \\
\hline & Pomar & 23 & 487 & 151 & 167 & 63 & 113 & 53 & 46 & 1103 & 53 & 2,249 & 7,422 & 0,5663 \\
\hline
\end{tabular}

(N) número de exemplares; (S) Número de espécies; (H') índice de Shannon-Wiener; ( $\alpha$ ) índice de Margalef; (E') índice de equitatividade. (Inv) Inverno, (Prim) Primavera, (Ver) Verão, (Out) Outono.

TABELA 4 - Valores de $t$ dos testes realizados para verificar diferença entre as árvores frutíferas no pomar do Colégio Agrícola Estadual Augusto Ribas, Ponta Grossa-PR (par a par) quanto ao índice de diversidade de Shannon-Wiener.

\begin{tabular}{ccccccccc} 
& Laranja & Limão & Poncã & Tangerina & Caqui & Maçã & Nectarina & Pera \\
\hline Limão & -1.02 & & & & & & & \\
Poncã & $-2.18^{*}$ & -1.32 & & & & & & \\
Tangerina & -0.19 & 0.90 & $2.13^{*}$ & & & & & \\
Caqui & $-3.00^{* *}$ & $-1.98^{*}$ & -0.30 & $-3.03^{* *}$ & & & & \\
Maçã & -0.36 & 0.52 & 1.61 & -0.21 & $2.15^{*}$ & & & \\
Nectarina & -1.36 & -0.21 & 1.27 & -1.25 & $2.06^{*}$ & -0.73 & & \\
Pera & 0.29 & 1.42 & $2.59^{*}$ & 0.52 & $3.64^{* * *}$ & 0.62 & 1.86 & \\
Pêssego & -0.29 & 0.79 & $2.03^{*}$ & -0.11 & $2.90^{* *}$ & 0.13 & 1.13 & -0.63 \\
$*$ p $<0.05 ; * * \mathrm{p}<0.01 ; * * *$ & $\mathrm{p}<0.001$ & & & & &
\end{tabular}




\section{CONCLUSÕES}

1-As subfamílias Eumolpinae e Galerucinae apresentaram maior abundância e riqueza de espécies entre os Chrysomelidae relacionados às frutíferas na região dos Campos Gerais do Paraná.

2-As plantas cítricas, juntamente com o caqui, apresentaram maior riqueza de crisomelídeos. Em termos de diversidade e equitabilidade, receberam destaque o caqui e a poncã.

3-A dificuldade na identificação das espécies e a ausência de trabalhos que tratem da fauna desta família, principalmente associada a árvores frutíferas, deixaram lacunas para uma completa compreensão das preferências alimentares destes insetos. Entretanto, os agrupamentos formados com base na estrutura da comunidade de crisomelídeos refletiram as características morfológicas das plantas.

4-Não houve correlação significativa da abundância com os fatores climáticos avaliados, mas a comunidade de crisomelídeos, associada com árvores frutíferas, exibiu elevada flutuação sazonal e interanual.

\section{AGRADECIMENTOS}

Ao professor Jail Bueno, diretor técnico do Colégio Agrícola Estadual Augusto Ribas, por permitir a entrada e as coletas nas áreas cultivadas. À doutoranda Patrícia Elizabeth Hüsch (Pós-Graduação em Entomologia, Universidade Federal do Paraná), pelo auxílio nas coletas. Ao administrador do Instituto Tecnológico, Sistema Meteorológico do Paraná, Osmar Stringari, por ceder os dados climáticos. À Regina Célia Botequio de Moraes (Depto. de Entomologia, Escola Superior de Agricultura "Luiz de Queiroz"), pelo envio do programa ANAFAU.

\section{REFERÊNCIAS}

BOUCHARD, P.; BOUSQUET, Y.; DAVIES, A.E.; ALONSO-ZARAZAGA, M.A.; LAWRENCE, J.F.; LYAL, C.H.C.; NEWTON, A.F.; REID, C.A.M.; SCHMITT, M. SLIPINSKI, A.; SMITH, A.B.T. Family-group names in Coleoptera (Insecta). ZooKeys, Sofia, v.88, p.1-972, 2011.

CANETTIERI, E.R.P.S.; GARCIA, A.H. Abundância relativa das espécies de Cerambycidae (Insecta-Coleoptera) em pomar de frutíferas misto. Pesquisa Agropecuária Tropical, Goiânia, v.30, n.2, p.43-50, 2000.
CHABOO, C.S. Biology and phylogeny of the Cassidinae Gyllenhal sensu lato (tortoise and leaf-mining beetles) (Coleoptera: Chrysomelidae). Bulletin of the American Museum of Natural History, New York, v.305, p.1-250, 2007.

COSTACURTA, N.C.; MARINONI, R.C.; CARVALHO, C.J.B. Fauna de Muscidae (Diptera) em três localidades do Estado do Paraná, Brasil, capturada por armadilha Malaise. Revista Brasileira de Entomologia, Curitiba, v.47, n.3, p.389-397, 2003.

GALLO, D.; NAKANO, O.; SILVEIRA NETO, S.; CARVALHO, R.P.L.; BAPTISTA, G.C.; BERTI FILHO, E.; PARRA, J.R.P.; ZUCCHI, R. A.; ALVES, S.B.; VENDRAMIM, J.D.; MARCHINI, L.C.; LOPES, J.R.S.; OMOTO, C. Entomologia agrícola. Piracicaba: FEALQ, 2002. 920p.

GONÇALVES, D. Levantamento das espécies e influência de variáveis climáticas sobre populações de Chrysomelidae. 2008. 87f. Dissertação (Mestrado) - Universidade Federal de Viçosa, Viçosa, 2008.

JOLIVET, P.; VERMA, K.K. Eumolpinae: a widely distributed and much diversified subfamily of leaf beetles (Coleoptera, Chrysomelidae). Terrestrial Arthropod Reviews, Leiden, v.1, p.3-37, 2008.

LINZMEIER, A.M.; RIBEIRO-COSTA, C.S. Seasonality and temporal structuration of Alticini community (Coleoptera, Chrysomelidae, Galerucinae) in the Araucaria Forest of Paraná, Brazil. Revista Brasileira de Entomologia, Curitiba, v.52, n.2, p.289-295, 2008.

LINZMEIER, A.M.; RIBEIRO-COSTA, C.S Spatial-temporal composition of Chrysomelidae (Insecta: Coleoptera) communities in southern Brazil. Journal of Natural History, London, v.46, p.1921-1938, 2012.

MARINONI, L.; MARINONI, R.C.; JORGE, C.M.; BONATTO, S.R. Espécies mais abundantes de Syrphidae (Diptera) em dois anos de coletas com armadilhas Malaise no Estado do Paraná, Brasil. Revista Brasileira de Zoologia, Curitiba, v.23, n.4, p.1071-1077, 2006. 
RILEY, E.G.; CLARK, S.M.; FLOWERS, R.W.; GILBERT, A.J. Chrysomelidae Latreille 1802. In: ARNETT JR, R.H.; THOMAS, M.C.; SKELLEY, P.E.; HOWARD, J.H. (Ed.). American beetles: Polyphaga: Scarabaeoidea through Curculionoidea. Boca Raton: CRC Press, 2002. v.2, p.617-691.
ZAWADNEAK, M.; ROSADO NETO, G.H.; SCHUBER, J.M.; PARCHEN, H.A. First Record of Spintherophyta semiaurata (Klug) (Coleoptera: Chrysomelidae) Damaging Strawberry Flowers. Neotropical Entomology, Londrina, v.40, n.3, p.407-408, 2011. 